\title{
Serine protease inhibitor, serpin, is a biomarker following Schistosoma mansoni re-infection in mice
}

\author{
Mohammed, E.S. ${ }^{{ }^{*}}$, El-Dakhly, Kh.M. ${ }^{2}$ \\ ${ }^{1}$ Department of Parasitology, Faculty of Veterinary Medicine, South Valley University, Qena 83522, Egypt \\ 2Department of Parasitology, Faculty of Veterinary Medicine, Beni-Suef University, Beni-Suef 62511, Egypt \\ *Corresponding author: emy.hosam@yahoo.com
}

\section{ARTICLE HISTORY}

Received: 13 October 2020

Revised: 3 February 2021

Accepted: 6 February 2021

Published: 30 April 2021

\begin{abstract}
Schistosomiasis is a chronic parasitic disease affecting mostly low income and resourcelimited countries. Despite the distribution of the curative medicine, praziquantel (PZQ), the frequency of re-infection is commonly reported, thus, making a difficulty to discriminate treatment failure after re-infection. Therefore, assessing Schistosoma mansoni re-infection after praziquantel administration is crucial to prove the treatment efficacy and to break the transmission of infection in endemic areas. The evolution of highly sensitive and specific diagnostic markers, reliable to detect the re-infection and to evaluate the treatment efficacy, is required to control schistosomiasis. In this study, the potential role of serpin recombinant antigen of S. mansoni as a biomarker of re-infection and chemotherapeutic efficacy has been assessed. Therefore, 20 mice were experimentally challenged and re-challenged with 50 S. mansoni cercariae and divided into 4 equal groups; the first included infected mice (control positive), the second group was twice infected with S. mansoni and left untreated, the third included mice twice infected then treated with praziquantel following the last challenge, and the forth one remained uninfected and untreated (control negative). The current findings demonstrated that high levels of IgG and IgG1 bound to serpin were detected following the re-infection and rapidly declined post treatment. In summary, S. mansoni recombinant serpin could be used as a promising marker to discriminate $S$. mansoni re-infection and evaluated the efficacy of treatment. The translation of such a potential tool in endemic areas will provide a significant support for the elimination and control programs against schistosomiasis.
\end{abstract}

Keywords: Schistosoma mansoni, serpin, praziquantel, re-infection, antibody, ELISA.

\section{INTRODUCTION}

Schistosomiasis is a chronic helminthic disease caused by several species belonging to the genus Schistosoma. Humans and other mammalian hosts acquire the infection via freshwater snails, the potential intermediate host (Colley et al., 2014). It is widely distributed in low income or limitedresources developing countries; however, it is prevalent in 76 countries (Steinmann et al., 2006; Chadeka et al., 2019). Despite of chemotherapeutic intervention and associated control measures, the disease is still prevalent with a potential risk of re-emerging (King, 2009). So far, praziquantel is the drug of choice, and extensively recommended by the mass drug administration (MDA) for the elimination of schistosomiasis (Cioli \& Pica-Mattoccia, 2003). However, reinfections with the active transmission have been noted, together with a raised evidence of the potential development of the resistance to praziquantel (Wang et al., 2012). In these circumstances, treatment failure/re-infection may be confused with the prior infection.
The life cycle of schistosomes comprises two hosts; mammals including humans, the definitive host, and freshwater snails, the intermediate host. In the later, the development of larval stages; miracidia, sporocysts, daughter sporocysts and furcocercus cercariae takes place. Cercariae penetrate human skin, forming schistosomulae that migrate through the blood circulation to pelvic/mesenteric venous plexuses where they grow into adult worms (Gurarie et al., 2018).

Coprological examination and serological/molecular tools are routine methods to detect $S$. mansoni re-infection. Basically, conventional tools, like Kato-Katz assay have been used for the purpose of fecal egg count with the possibility of detecting various helminth eggs, however, they could not detect prepatent infections before egg release in stools, therefore, they are less sensitive in districts with low prevalences (Berhe et al., 2004; McCarthy et al., 2012).

Molecular assays like PCR have been evoked for the accurate detection of schistosomiasis. Those tools could detect DNA and parasite-free DNA in biological human 
samples (feces, urine, sera, plasma) as well asin snails (Pontes et al., 2002; Sandoval et al., 2006; Wichmann et al., 2009; Fernández-Soto et al., 2014; Aboelhadid et al., 2016). Despite the increased and required degree of specificity and sensitivity, their use in endemic areas has various limitations like unaffordable cost, DNA preparation processes, applicability in field conditions and the requirement for welltrained personnel.

Moreover, the urine-based point of care assay (POC-CCA) was used to detect circulating antigen of living worms in urine samples providing valuable findings in terms of diagnosis and treatment. Such assay is convenient and efficient compared to the KK test due to easy and rapid performance (Greter et al., 2016). However, more studies are required to assess the field sensitivity of this technique, particularly in less endemic areas (Stothard et al., 2006; Lodh et al., 2013; Adriko et al., 2014). On the other hand, the serological assays using $S$. mansoni crude antigen derived from Schistosoma adult worms/eggs have been used to overcome obstacles revealed by conventional parasitological tests. Although this sensitivity, they have a drawback of cross-reactivity with other helminths. In addition, these immunodiagnostic tests could not be used to evaluate the efficacy of treatment since specific antibodies might be detected in blood several months post treatment (Zhu et al., 2005).

As a result of the risk of species cross-reactivity as well as failure of the antibody detection using the crude antigens in distinguishing the persisting infection from past one, as the occurrence of antibodies even after cure is considerably responsible for the false positive infections (Doenhoff et al., 2004; Hinz et al., 2017), the use of S. mansoni recombinant antigen might be greatly useful to detect parasite-specific antibodies and, thus, to determine the actual prevalence of schistosomiasis in low prevalence-endemic areas (Hamilton et al., 1998). Furthermore, these limitations could be minimized by the proper selection of specific recombinant antigens to induce species-specific and shortlived antibodies in response to the parasite infection, thus, antibodies should be strongly sensitive to chemotherapy or consecutive exposure (Mohammed et al., 2020).

Serine protease inhibitor (serpin) recombinant antigen of $S$. mansoni has been reported a promising speciesspecific diagnostic antigen for diagnosis of schistosomiasis (Tanigawa et al., 2015). It had a strong reactivity to sera of $S$. mansoni-infected patients, and with sera from experimentally infected rats as well (Mohammed et al., 2020). Since antibodies to recombinant serpin are species-specific and they are elevated at the early stage of experimental S. mansoni infection, the present study aimed to determine the potential use of such antigen to monitor the re-infection with Schistosoma spp. and evaluate the treatment efficacy of praziquantel in mice experimentally infected with schistosomes.

\section{MATERIALS AND METHODS}

\section{Parasite culture}

S. mansoni Puerto Rican strain has been used. In the animal facility building at Nagasaki University, Japan, the life cycle of the parasite was done by the successive passage of the parasite in Biomphalaria glabrata snails and ICR mice. Each mouse was percutaneously infected with 250 cercariae and sacrificed 7 weeks after infection. Following cardiac perfusion with phosphate buffer saline, adult worms are extracted from the portal vein and eggs are isolated from the livers of infected mice (Mohammed et al., 2020).

\section{Animals and experimental design}

Female C57BL/6 mice aged 28 weeks were purchased from Japan SLC Inc. (Shizuoka, Japan) and they are kept under hygienic and strict pathogen-free conditions. Animal housing, handling and control were carried out in accordance with the Nagasaki University agreement. The experimental design was approved by the Nagasaki University Ethical Committee (approval number 1505181226) and was carried out in compliance with the animal facilities guidelines in Nagasaki University and Japanese law for Humane Treatment and Management of Animals.

Experimentally, mice were divided into 4 equal groups: Mice in the first group (G1) were infected with S. mansoni cercariae and left untreated (control positive), mice of the second group (G2) were re-infected and left untreated, and animals of the third group (G3) were re-infected and treated with PZQ (re-infected treated group). For the re-infection, mice of both G2 and G3 were re-challenged with 50 S. mansoni cercariae in the inguinal area 9 weeks following the first infection. In G3, mice were first treated at 8 weeks post infection with PZQ (Sigma, St. Louis, MO) $500 \mathrm{mg} / \mathrm{kg}$ body weight twice for two consecutive days, then treated again 5 weeks post re-infection (14 weeks post the primary infection). PZQ was dissolved in distilled water and $230 \mu \mathrm{l}$ of the solution was administered orally by gavage. To estimate the worm burden, one mouse was sacrificed one week post treatment ( 9 weeks post infection), and adult worms were checked in the portal vein via perfusion. All mice were sacrificed at the end of the experiment ( 18 weeks post infection) and the effectiveness of the second treatment was assessed. Uninfected mice of the fourth group (G4) were kept untreated and used as a negative control.

\section{Collection of sera}

Blood samples were collected from mice of all groups via the tail vein biweekly until 18 weeks post infection. They were allowed to coagulate at room temperature for 2 hours at $1000 \times \mathrm{g}$ for $10 \mathrm{~min}$ at $4^{\circ} \mathrm{C}$ before centrifugation. Serum samples were collected and processed in Eppendorf tubes at $-30^{\circ} \mathrm{C}$ before their use (Kalenda et al., 2015).

\section{Preparation of recombinant protein}

Serine protease inhibitor (serpin) was prepared as previously described by Tanigawa et al. (2015) through a collaborative work. The protein was expressed using bacterial expression system as a His-tagged protein at $\mathrm{N}$-terminal.

\section{Detection of antibodies using ELISA}

In order to detect specific antibodies against serpin antigens, enzyme-linked immunosorbent assay (ELISA) was performed on mice sera. Nunc MaxiSorp 96 wells (Nalgen Nuncint, Roskilde, Denmark) microtiter plates were coated with $50 \mu \mathrm{l}$ per well of antigen solution ( $5 \mu \mathrm{g} / \mathrm{ml}$ ) (PBS) of recombinant antigen overnight. Plates were washed 3 times at room temperature with PBS containing $0.05 \%$ Tween 20 (PBST), then blocked for 2 hours at room temperature with 1\% bovine serum albumin (BSA) (Sigma) in PBS. Plates were washed with (PBST) and filled with mice sera that were diluted 50 times in PBST containing $0.1 \%$ BSA and incubated at room temperature for 2 hours. After washing as mentioned above, the plate was incubated with horseradish peroxidase (HRP)conjugated goat anti-mouse IgG antibody (R\&D Systems, Minneapolis, MN) diluted in PBST 1:1000 containing 0.1\% BSA for 1 hour. For anti-mouse IgG1-HRP (Invitrogen) antibodies were used in PBST containing $0.1 \%$ BSA at $1: 1000$ dilution. The plates were cleaned, accompanied by the addition of tetramethylbenzidine (TMB) colorimetric substrate ( $B D$ 
pharmingen, Allschwil, Switzerland). Using the Multiskan FC microplate reader (Thermo Scientific), absorbance was determined at $450 \mathrm{~nm}$. Duplicate serum samples were tested. Control negative sera on each ELISA plate were also included. This method was applied according to (Kalenda et al., 2015; Mohammed et al., 2020).

\section{Statistical analysis}

For statistical analysis and graph creation, GraphPad Prism 5.0 (GraphPad Software Inc., La Jolla, CA) and Excel software are used. The ELISA cut-off values for infected mice were determined as the mean plus three normal optical density (OD) deviations (mean + 3SD) of the control group serum. OD readings equal to or less than the cut-off value were considered negative, while those were considered negative, whereas such readings were considered positive above the cut-off value. For typically distributed samples, ANOVA and a $t$ test were used and the Mann-Whitney test was used for the other non-normally distributed samples. $P<0.05$ was deemed statistically significant when comparing variations between groups (Kalenda et al., 2015).

\section{RESULTS AND DISCUSSION}

Dynamics of IgG and IgG1 subtype reacting with serpin during Schistosoma mansoni re-infection

Currently, levels of both anti-serpin IgG and IgG1 significantly $(P \leq 0.01)$ increased one week post re-infection (from 10 weeks) in sera of mice of the re-infected group compared to those detected in the infected group. This increase was maintained during the re-infection (Figure $1 \mathrm{a}, \mathrm{c}$ ) suggesting that the level of specific IgG1 antibodies bound to serpin could distinguish re-infected mice from firstly infected ones. Such observation was consistent with Demeure et al. (1993) who found that a higher production of IgG4 (in human sera) or IgG1 (in mice) is correlated with the re-infection. Previous immunological studies of $S$. mansoni infection have been shown a higher level of IgG4 correlating with S. mansoni

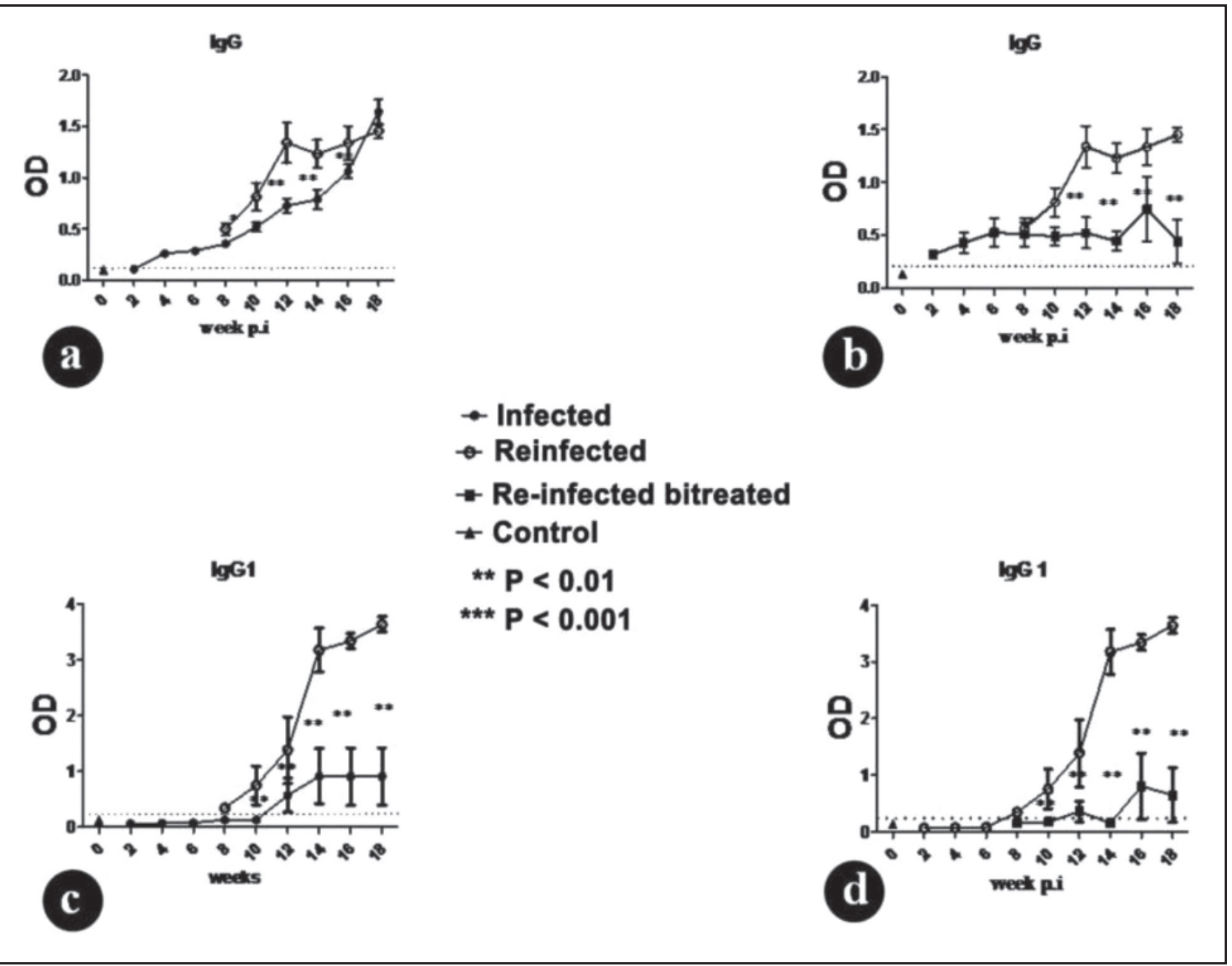

Figure 1. Total serum IgG and IgG1 responses to recombinant $S$. mansoni antigens serpin during $S$. mansoni infection, re-infection and treatment. Sera from five mice were used at each time point. Data are presented as graphs, and means are shown with the standard errors. The dashed line represents the cut-off value (mean + 3SD of the OD of the uninfected controlgroup). a, c The total IgG and IgG1 levels at each time point in the period 2-18 weeks post-infection for infected group untreated (black circle) compared to that in the re-infected untreated group (white circle). b, d The total IgG and IgG1 levels at each time point in the period 2-18 weeks post-infection forre-infected untreated group (white circle) compared to that in the re-infected treated group (black square). Black triangle represented mice of the uninfected control group. ${ }^{* *} P<0.01$ and $* * * P<0.001$. 
re-infection, rendering IgG4 antibody subtype a useful biomarker of parasitic re-infections, particularly schistosomiasis (Sulbarán et al., 2010; Tanigawa et al., 2015; Mohammed et al., 2020). It has been found that serpin could be used as a diagnostic tool for the early detection of $S$. mansoni infection. Additionally, serpin of $S$. mansoni had a significantly higher reactivity to sera from $S$. mansoni-infected rats and patients suggesting that the immunodiagnostic potential of serpin arises from the important physiological role of host-parasite interactions that induce host immunity on releasing the host tissue (Smithers \& Terry, 1965; Molehin et al., 2014; Mohammed et al., 2020).

Experimentally, it has been that $\operatorname{lgG} 1$ in mice is equivalent to IgG4 in humans, therefore, evaluating the level of mice IgG1 would reflect comparable information as for human IgG4 (Lilienthal et al., 2018). Sera of infected and reinfected mice were probed against serpin recombinant antigen derived from Schistosoma mansoni to detect IgG and $\lg G 1$.

Dynamics of IgG and IgG1 subtype reacting with serpin during Schistosoma mansoni re-infection and treatment with PZQ

So far, few numbers of efficient markers to predict cure or reinfection have been reported. To evaluate the efficacy of treatment against the re-infection on kinetics of both IgG and IgG1 bound to serpin recombinant antigen, sera from re-infected untreated and re-infected bitreated mice group were assayed by ELISA. Levels of both IgG and IgG1 reacting to serpin were significantly declined $(P \leq 0.01)$ one week after treatment with re-infection compared to the re-infection without treatment (Figure $1 b, d$ ). The decreased level of specific antibodies to serpin following treatment suggests that IgG and IgG1 against serpin are highly susceptible to treatment and correlate with parasite clearance in the treated group.

It is worthy to mention that the specific IgG and IgGI rapidly declined post treatment with the re-infection. This finding might suggest that $S$. mansoni serpin induced a specific and short-lived antibody response which often concomitant with the persistence of the corresponding pathogenic antigen in vivo (Wang et al., 2013). Previous literature proposed that evaluating short-lived antigen-dependent antibodies could be useful for the detection of the parasite as well as to properly evaluate the drug efficacy (Alderete et al., 1991; Zang et al., 2000).

The selection of target antigens is crucial and dependant on the diagnosis of the stage of the disease. For the diagnostic purpose, it could be helpful to select for an antigen that is expressed in all stages of the parasite life cycle inside the definitive host (schistosomulum, adult worm and egg) whereas the selection of antigens should emphasize on the adult worm expressed protein, excluding proteins that are abundantly expressed in the egg stage.

Indeed, following a successful treatment with PZQ, adult schistosomes as well as their antigens are eliminated; however, eggs remain trapped in the liver and intestinal tissues for seven months after a complete cure. The eggreleased antigens are able to maintain a persistent antibody production (Traggiai et al., 2003). Concomitantly, further works are mandatory to diagnostically use serpin as a biomarker in endemic areas where a cohort of subjects will be evaluated before PZQ and periodically afterwards to assess the treatment efficacy as well as re-infection. Additionally, samples from infected persons who visited endemic areas might be helpful as a single infection. The translation of such a potential tool in endemic areas will provide a significant support for the elimination and control programs against schistosomiasis.

\section{CONCLUSION}

This study has shown that the recombinant antigen (serpin) is likely be potential for guiding re-infection and efficacy of chemotherapeutic treatment against schistosomiasis.

\section{ACKNOWLEDGMENT}

We thank to Department of Eco-Epidemiology and Department of Parasitology, Institute of Tropical Medicine, Nagasaki University, Japan for providing recombinant antigen and mice beside to their help and support to conduct this study. The experimental design was approved by the ethical committee of Nagasaki University, Japan.

\section{Conflicts of interest}

None.

\section{REFERENCES}

Aboelhadid, S.M., Thabet, M., El-Basel, D. \& Taha, R. (2016). Digenetic larvae in Schistosome snails from El Fayoum, Egypt with detection of Schistosoma mansoni in the snail by PCR. Journal of Parasitic Diseases 40: 730-734. https://doi. org/10.1007/s12639-014-0567-7

Adriko, M., Standley, C.J., Tinkitina, B., Tukahebwa. E.M., Fenwick, A., Fleming, F.M., Sousa-Figueiredo, J.C., Stothard, J.R. \& Kabatereine, N.B. (2014). Evaluation of circulating cathodic antigen (CCA) urine-cassette assay as a survey tool for Schistosoma mansoni in different transmission settings within Bugiri District. Uganda. Acta Tropica 136: 50-57. https://doi.org/10.1016/j.actatropica.2014.04.001

Alderete, J.F., Newton, E., Dennis, C., Engbring, J. \& Neale, K.A. (1991). Vaginal antibody of patients with trichomoniasis is to a prominent surface immunogen of Trichomonas vaginalis. Genitourinary Medicine 67: 220-225. https://doi.org/ 10.1136/sti.67.3.220

Berhe, N., Medhin, G., Erko, B., Smith, T., Gedamu, S., Bereded, D., Moore, R., Habte, E., Redda, A. \& Gebre-Michael, T. (2004). Variations in helminth faecal egg counts in KatoKatz thick smears and their implications in assessing infection status with Schistosoma mansoni. Acta Tropica 92: 205-212. http://doi.org/10.1016/j.actatropica.2004.06.011

Chadeka, E.A., Nagi, S., Cheruiyot, N.B., Bahati, F., Sunahara, T., Njenga, S.M. \& Hamano, S. (2019). A high-intensity cluster of Schistosoma mansoni infection around Mbita causeway, western Kenya: a confirmatory crosssectional survey. Tropical Medicine and Health 47: 26. https://doi.org/ 10.1186/s41182-019-0152-y

Cioli, D. \& Pica-Mattoccia, L. (2003). Praziquantel. Parasitology Research 90: S3-S9. http://doi.org/10.1007/s00436-0020751-z

Colley, D.G., Bustinduy, A.L., Secor, W.E. \& King, C.H. (2014). Human schistosomiasis. The Lancet 383: 2253-2264. https://doi.org/10.1016/S0140-6736 (13)61949-2

Demeure, C.E., Rihet, P., Abel, L., Ouattara, M., Bourgois, A. \& Dessein, A.J. (1993). Resistance to Schistosoma mansoni in humans: influence of the IgE/IgG4 balance and IgG2 in immunity to reinfection after chemotherapy. The Journal of Infectious Diseases 168: 1000-1008. https://doi.org/ 10.1093/infdis/168.4.1000 
Doenhoff, M.J., Chiodini, P.L. \& Hamilton, J.V. (2004). Specific and sensitive diagnosis of schistosome infection: can it be done with antibodies? Trends in Parasitology 20: 35-39. https://doi.org/10.1016/j.pt.2003.10.019

Fernández-Soto, P., Gandasegui Arahuetes, J., Sánchez Hernández, A., López Abán, J., Vicente Santiago, B. \& Muro, A. (2014). A loop-mediated isothermal amplification (LAMP) assay for early detection of Schistosoma mansoni in stool samples: a diagnostic approach in a murine model. PLoS Neglected Tropical Diseases 8. e3126. http://doi.org/ 10.1371/journal.pntd.0003126

Greter, H., Stefanie, J.K., Bongo, N.R.N., Idriss, O.A., Jakob, Z. \& Jürg, U. (2016). Validation of a point-of-care circulating cathodic antigen urine cassette test for Schistosoma mansoni diagnosis in the Sahel, and potential crossreaction in pregnancy. American Journal of Tropical Medicine and Hygiene 94: 361-364. http://doi.org/10.4269/ajtmh.150577

Gurarie, D., Lo, N.C., Ndeffo-Mbah, M.L., Durham, D.P. \& King, C.H. (2018). The human snail transmission environment shapes long term schistosomiasis control outcomes: Implications for improving the accuracy of predictive modeling. PloS Neglected Tropical Diseases 12: e0006514. http://doi.org/10.1371/journal.pntd.0006514

Hamilton, J.V., Klinkert, M. \& Doenhoff, M.J. (1998). Diagnosis of schistosomiasis: antibody detection, with notes on parasitological and antigen detection methods. Parasitology 17: 41-57. https://doi.org/10.1017/S0031182099004205

Hinz, R., Schwarz, N.G., Hahn, A. \& Frickmann, H. (2017). Serological approaches for the diagnosis of schistosomiasis - A review. Molecular and cellular Probes 31: 2-21. https://doi.org/10.1016/j.mcp.2016.12.003

Kalenda, Y.D.J., Kato, K., Goto, Y., Fujii, Y. \& Hamano, S. (2015). Tandem repeat recombinant proteins as potential antigens for the sero-diagnosis of Schistosoma mansoni infection. Parasitology International 64: 503-512. https:// doi.org/10.1016/j.parint.2015.06.012

King, C.H. (2009). Toward the elimination of schistosomiasis. New England Journal of Medicine 360: 106-109. https:// doi.org/10.1056/NEJMp0808041

Lilienthal, G.M., Johann, R., Janina, P., Yannic, C.B., Alexei, L. \& Marc, E. (2018). Potential of murine IgG1 and human IgG4 to inhibit the classical complement and Fcy receptor activation pathways. Frontiers in Immunology 9: 958. https://doi.org/10.3389/fimmu.2018.00958.

Lodh, N., Mwansa, J.C., Mutengo, M.M. \& Shiff, C.J. (2013). Diagnosis of Schistosoma mansoni without the stool: comparison of three diagnostic tests to detect Schistosoma mansoni infection from filtered urine in Zambia. American Journal of Tropical Medicine and Hygiene 89: 46-50. https:// doi.org/10.4269/ajtmh.13-0104

McCarthy, J.S., Sara, L., Guo-Jing, Y., Rashida, M.B., Héctor, H.G., Banchob, S., Arve, L.W., Roger, K.P. \& María-Gloria, B. (2012). A research agenda for helminth diseases of humans: diagnostics for control and elimination programmes. PLOS Neglected Tropical. Diseases 6: e1601. https://doi.org/ 10.1371/journal.pntd.0001601

Mohammed, E.S., Nakamura, R., Kalenda, Y.D.J., Deloer, S., Moriyasu, T., Tanaka, M., Fujii, Y., Kaneko, S., Hirayama, K. \& Ibrahim, A.I. (2020). Dynamics of serological responses to defined recombinant proteins during Schistosoma mansoni infection in mice before and after the treatment with praziquantel. PLoS Neglected Tropical Diseases 14: e0008518. https://doi.org/10.1371/journal.pntd.0008518.

Molehin, A.J., Geoffrey, N.G., Patrick, D. \& Donald, P.M. (2014). Characterisation of a secretory serine protease inhibitor (SjB6) from Schistosoma japonicum. Parasites \& Vectors 7: 330. https://doi.org/10.1186/1756-3305-7-330
Pontes, L.A., Dias-Neto, E. \& Rabello, A. (2002). Detection by polymerase chain reaction of Schistosoma mansoni DNA in human serum and feces. American Journal of Tropical Medicine and Hygiene 66: 157-162. https://doi.org/10.4269/ ajtmh.2002.66.157

Sandoval, N., Siles-Lucas, M., Pérez-Arellano, J.L., Carranza, C., Puente, S., López-Abán, J. \& Muro, A. (2006). A new PCRbased approach for the specific amplification of DNA from different Schistosoma species applicable to human urine samples. Parasitology 133: 581-587. https://doi.org/ 10.1017/S0031182006000898

Smithers, S.R. \& Terry, R.J. (1965). The infection of laboratory hosts with cercariae of Schistosoma mansoni and the recovery of the adult worms. Parasitology 55: 695-700. https://doi.org/10.1017/S0031182000086248

Steinmann, P., Keiser, J., Bos, R., Tanner, M. \& Utzinger, J. (2006). Schistosomiasis and water resources development: systematic review, meta-analysis, and estimates of people at risk. Lancet Infectious Diseases 6: 411-425. https://doi.org/10.1016/S1473-3099(06)70521-7

Stothard, J.R., Kabatereine, N.B., Tukahebwa, E.M., Kazibwe, F., Rollinson, D., Mathieson, W., Webster, J.P. \& Fenwick, A. (2006). Use of circulating cathodic antigen (CCA) dipsticks for detection of intestinal and urinary schistosomiasis. Acta Tropica 97: 219-228. https://doi.org/10.1016/ j.actatropica.2005.11.004

Sulbarán, G.S., Ballen, D.E., Bermúdez, H., Lorenzo. M., Noya, O. \& Cesari, I.M. (2010). Detection of the Sm31 antigen in sera of Schistosoma mansoni-infected patients from a low endemic area. Parasite Immunology 32: 20-28. https:// doi.org/10.1111/j.1365-3024.2009.01152.x

Tanigawa, C., Fujii, Y., Miura, M., Nzou, S.M., Mwangi, A.W., Nagi, S., Hamano, S., Njenga, S.M., Evaristus, C.M. \& Hirayama, K. (2015). Species-specific serological detection for schistosomiasis by serine protease inhibitor (SERPIN) in multiplex assay. PLoS Neglected Tropical Diseases 9: e0004021. https://doi.org/10.1371/journal.pntd.0004021

Traggiai, E., Puzone, R. \& Lanzavecchia, A. (2003). Antigen dependent and independent mechanisms that sustain serum antibody levels. Vaccine 21: 35-37. https://doi.org/ 10.1016/S0264-410X(03)00198-1

Wang, W., Wang, L. \& Liang, Y.S. (2012). Susceptibility or resistance of praziquantel in human schistosomiasis: a review. Parasitology Research 111: 1871-1877. https:// doi.org/10.1007/s00436-012-3151-z

Wang, J., Zhao, F., Yu, C., Xiao, D., Song, L.J., Yin, X.R., Shen, S., Hua, W.Q., Zhang, J.Z. \& Zhang, H.F. (2013). Identification of proteins inducing short-lived antibody responses from excreted/secretory products of Schistosoma japonicum adult worms by immunoproteomic analysis. Journal of Proteomics 87: 53-67. https://doi.org/10.1016/j.jprot.2013.05.003

Wichmann, D., Marcus, P., Thomas, Q., Stefanie, K., Gerd-Dieter, B., Christoph, G. \& Christian, D. (2009). Diagnosing schistosomiasis by detection of cell-free parasite DNA in human plasma. PLoS Neglected Tropical Diseases 3: e422. https://doi.org/10.1371/journal.pntd.0000422

Zang, X., Atmadja, A.K., Gray, P., Allen, J.E., Gray, C.A., Lawrence, R.A., Yazdanbakhsh, M. \& Maizels, R.M. (2000). The serpin secreted by Brugia malayi microfilariae, Bm-SPN-2, elicits strong, but short-lived, immune responses in mice and humans. The Journal of Immunology 165: 5161-5169. https://doi.org/10.4049/jimmunol.165.9.5161

Zhu, Y.C., Socheat, D., Bounlu, K., Liang, Y.S., Sinuon, M., Insisiengmay, S., He, W., Xu, M., Shi, W.Z. \& Bergquist, R. (2005). Application of dipstick dye immunoassay (DDIA) kit for the diagnosis of schistosomiasis mekongi. Acta Tropica 96: 137-141. https://doi.org/10.1016/j.actatropica. 2005.07.008 